\title{
Les jeux d'enfants dans l'oasis de Tabalbala
}

(Sahara nord-occidental - Algérie)

D. Champault

\section{(2) OpenEdition}

\section{Journals}

Édition électronique

URL : http://journals.openedition.org/encyclopedieberbere/1513

DOI : $10.4000 /$ encyclopedieberbere.1513

ISSN : 2262-7197

Éditeur

Peeters Publishers

Édition imprimée

Date de publication : 1 septembre 2003

Pagination : 3892-3895

ISBN : 2-7449-0424-4

ISSN : 1015-7344

Référence électronique

D. Champault, « Les jeux d'enfants dans l'oasis de Tabalbala », Encyclopédie berbère [En ligne], 25 | 2003, document J10c, mis en ligne le 01 juin 2011, consulté le 25 septembre 2020. URL : http:// journals.openedition.org/encyclopedieberbere/1513; DOI : https://doi.org/10.4000/ encyclopedieberbere.1513

Ce document a été généré automatiquement le 25 septembre 2020

(c) Tous droits réservés 


\title{
Les jeux d'enfants dans l'oasis de Tabalbala
}

\author{
(Sahara nord-occidental - Algérie) \\ D. Champault
}

\section{NOTE DE L'ÉDITEUR}

(d'après D. CHAMPAULT, Une oasis du Sahara nord-occidental, Tabelbala, Paris, CNRS, 1969)

\section{Le camion et le pain}

1 Tous les garçons rêvent de l'exceptionnelle promotion sociale du chauffeur de camion. Deux types se partagent la faveur des petits garçons :

- L'ancien bidon de pétrole, muni de 4 roues de poterie grossièrement cuite, pourvu d'une roue de secours.

- Les boîtes d'eau d'Évian sont à trouées de part en part pour le passage d'un fil de fer : elles serviront respectivement de pont avant et de pont arrière. Tout un système compliqué de fils de fer réunit les deux ponts et forme une sorte de superstructure sommaire qui représente la carrosserie.

Camion-bidon et camion-boîtes sont utilisés de la même façon : courses ferraillantes sur les cailloux : ce qui est important alors, est de faire le maximum de bruit.

«Faire le pain » est la préoccupation la plus fréquente, peut-être par simple projection du souci permanent des enfants qui partent le matin ventre vide et n'ont pour passer tout un jour que quelques dattes, très exceptionnellement un petit pain aux oignons.

4 Les rondelles obtenues sont baptisées "pains ». Elles peuvent être perforées en leur centre : elles s'appelleront alors poulies de puits et joueront sur un axe, léger morceau de bois que l'on posera sur deux montants mortaises en bois ou en poterie. 
Le mobilier de la maison : marmite, pots, assiettes, verres, tout matériel indispensable à la dînette, sera figuré par des tessons de bouteille ou de poterie.

Les maisons et leur mobilier sont abandonnés par leurs propriétaires qui les retrouveront intacts plusieurs mois après. Les propriétaires consciencieux prennent d'ailleurs la précaution d'enclore leurs maisons d'un grand cercle tracé avec le pied.

\section{La ferfara (Rhombe) (filles et garçons)}

7 La ferfara en usage à Tabelbala, malgré l'identité de nom, ne ressemble pas à celle décrite par Doutté chez les Rehamna du Maroc : « planchette longue et mince à laquelle on attache une cordelette de $1 \mathrm{~m}$ à $1,50 \mathrm{~m}$, que l'on tord en tenant la planchette immobile. On lâche le tout et on fait tourner l'instrument à la manière d'une fronde qui produit une sorte de sifflement intermittent ».

8 La ferfara ("Rhombe ») possède un couvercle de boîtes de conserves, carton fort, poterie, ou bois de récupération, percé en son milieu de deux trous très rapprochés. Le diamètre du disque peut varier de 3 à $15 \mathrm{~cm}$. Une cordelette de laine passe par les deux perforations et, une fois nouée, forme deux boucles de part et d'autre du disque. La longueur de chaque bouche est d'environ $20 \mathrm{~cm}$. L'enfant tord légèrement la cordelette, passe un index dans chaque boucle et, par un mouvement de va-et-vient alternatif, fait tourner le disque qui, bientôt, prend une certaine vitesse et produit un ronflement caractéristique. Ce jouet très populaire est utilisé indifféremment par les filles et par les garçons. Il a des virtuoses, capables de faire tourner et ronfler, au milieu d'une cordelette de $1,20 \mathrm{~m}$, une minuscule rondelle en test d'œuf d'autruche.

\section{Le jeu des maisons}

9 Tous les enfants de garde au pâturage, filles ou garçons, compensent leur éloignement en recréant un village à leur échelle : c'est le jeu des « maisons » (gayu n-hayu).

10 La forme la plus classique de ce jeu comporte la disposition de petits cailloux selon le plan fantaisiste d'un village où chaque maison couvre au maximum un mètre carré mais comporte des aménagements plus fastueux que ceux des habitations réelles. Tout village est pourvu de sa mosquée et de plusieurs puits.

11 Aux garçons revient le soin d'équiper le puits, de creuser l'abreuvoir, de tresser les seaux.

12 Les filles simuleront des récipients, feront des magasins, des fours, des pains. Les matériaux en sont à leur disposition : ce sont par ordre de fréquence d'utilisation, des tessons de poterie anciens, parfois néolithiques (la poterie de Kuka Ayaš, la poterie actuelle, ne vaut rien, disent les enfants), des fragments de test d'œuf d'autruche, des brindilles de bois, des tiges de graminées utilisées pour des vanneries miniatures.

\section{Les jouets vivants}

13 Garçons et filles ont l'occasion d'avoir des jouets vivants : ce sont les asenkri, scinques dits «poissons de sable» (Scincus officinalis), les agerzem (Uromastix acanthinurus), les fennecs (Fennecus zerda). Leurs pères allant chasser, ou partant à la quête de terfes ou de 
graminées, ne manquent pas de suivre les traces de tout animal qu'ils trouvent sur leur chemin.

Les scinques auront les pattes brisées pour qu'ils ne puissent s'échapper.

La morsure de l'Uromastix est évitée en lui cousant les commissures avec deux fibres de palmier.

Les enfants peuvent organiser de véritables courses entre leurs agerzem respectifs comme s'il s'agissant d'une course de chevaux.

Pour activer leurs coursiers, ils prennent une longue baguette flexible et en grattent le dos des lézards. Celui dont l'agerzem court le plus vite et arrive le premier à un but désigné au préalable, est le gagnant.

Les scinques peuvent être traités comme de véritables poupées, habillés de chiffons et dorlotés.

Qu'ils s'agissent d'Uromastix ou de Scincus, le jeu se termine de la même façon. Avant de succomber à ces divers traitements de faveur, dès qu'ils donnent des signes d'épuisement, ils sont égorgés par l'homme qui se trouve à portée, puis mangés par les enfants.

\section{Le jeu de Kwara}

Les joueurs 8,10 ou 12 sont répartis en deux camps, camp de l'est, camp de l'ouest. Les buts assignés sont en arrière de la ligne de chaque parti. La balle, tiza, doit être poussée exclusivement à la crosse, jusqu'au but du camp adverse. La crosse est constituée par le pédoncule spatule d'une grosse palme réduite à environ $70 \mathrm{~cm}$.

est mise en jeu au milieu du terrain par un tiers non engagé dans la partie. Les points sont comptés par les spectateurs et par les joueurs eux-mêmes. Il faut obtenir cinq points pour gagner une partie.

Lorsque les joueurs sont des enfants, les vainqueurs montent sur le dos des vaincus, se partagent en deux camps et engagent une partie de kwara montée.

Les enfants jouent à la kwara particulièrement en hiver et en automne, mais à Tabelbala, il ne faut pas voir, dans le choix, une détermination rigoureuse.

Par tradition, au lendemain d'un jour de pluie, les hommes célibataires s'affrontent en deux camps en un jeu de kwara qui diffère de celui des enfants par la nature de la crosse et celle de la balle.

Pour cette occasion, ils retrouvent une crosse en bois d'éthel, ou de jujubier, ou la confectionnent en bois vert, mis à chauffer dans un feu d'herbes ou de folioles de palmier, et maintenu en position recourbée au moyen d'une quelconque ficelle jusqu'au moment de l'emploi.

21 Le plus souvent, la balle n'est plus la tiza déjà décrite, mais la rqaba, c'est-à-dire rotule de chameau légèrement façonnée ; une tête de fémur de chameau peut être également façonnée, mais, bien que plus régulièrement sphérique, on lui préfère une rotule plus solide et qui n'éclate pas sous les vigoureux coups de crosse qui la propulsent comme un palet plutôt que comme une balle.

22 Auparavant les hommes mariés s'affrontaient à la kwara. Il y aurait eu des parties réservées aux hommes mûrs, d'autres aux célibataires. Les vieillards eux-mêmes n'auraient pas dédaigné d'organiser leur propre jeu. La kwara se pratiquait alors non 
pas au lendemain de la pluie, mais le jour même où les jeunes filles promenaient tarenža dans le village, et qu'alors les actions conjuguées des hommes et des femmes n'auraient pas manqué d'« ouvrir » le ciel.

Actuellement la kwara ne semble pas liée à l'obtention de la pluie comme elle le fut longtemps en d'autres points du Maghreb. Seule l'orientation du jeu est strictement respectée.

\section{Le coup de pied de l'âne (jeu de garçons)}

On assigne une place à l'enfant qui doit faire l'âne. Il fait dans le sable un trou peu profond et s'y tient sur le pied gauche.

Autour de lui les enfants forment un cercle assez serré et tournent épaule contre épaule, assez lentement, tout en excitant l'âne par des onomatopées diverses. L'âne, de son pied libre, rue et tâche de toucher un des enfants. Le premier touché par le coup de pied de l'âne sera l'âne à son tour.

\section{La muette (jeu de filles)}

Les enfants forment un cercle, s'assoient en tailleur, et, chacun à son tour, tape sur la cuisse de sa voisine de droite. Le jeu doit se faire dans le silence le plus parfait. La première qui prononce une parole ou qui se laisse aller à rire est exclue du jeu.

\section{Le jeu de la grand-mère et de l'ogresse (filles sans limitation d'âge ; garçons au-dessous de 8 ans)}

Deux fillettes de 10 à 12 ans mènent le jeu ; l'une sera la grand-mère, l'autre l'ogresse. Une ronde se forme et tourne (dans le sens des étoiles) en récitant des incantations. Puis les enfants s'agenouillent et font semblant de ramasser des dattes en rassemblant un petit tas de sable.

Chaque enfant doit annoncer une espèce de dattes différente; pendant ce temps la grand-mère et l'ogresse partent à l'écart.

Toutes deux placent entre leurs orteils du pied gauche et entre chaque doigt de leurs mains des brindilles ou des crottes de chèvre. Elles reviennent à cloche-pied vers les enfants toujours agenouillés qui font le geste de rouler le couscous.

La grand-mère et l'ogresse entrent dans le cercle en sautillant sur le pied droit.

Sans quitter leur place, les enfants doivent essayer d'attraper le maximum de viande, représentée par les bâtonnets et les crottes placés entre les doigts de l'ogresse et de la grand-mère.

Ceux qui auront réussi à subtiliser le plus grand nombre de brindilles et de crottes seront à leur tour ogresse et grand'mère. 


\section{Les jeux de siryu}

28 Il s'agit de plusieurs jeux utilisant des bâtonnets, longs de $25 \mathrm{~cm}$, taillés dans les stipes de palmier. L'un des deux joueurs choisit les bâtonnets, l'autre des cailloux. Ces siryu jouent, en fait, le rôle de dés. Ils sont lancés dans un damier tracé dans le sable, qui tient lieu de marelle. Il existe trois variétés de jeu. Les damiers comptent un nombre variable de cases. Les trois dernières sont Le Caire, Médine et la Mekke. A gagné la partie le joueur qui a conduit ses trois pièces à la Mekke.

INDEX

Mots-clés : Algérie, Ayt Mgild, Enfant, Ethnographie, Kabylie, Moyen-Atlas, Sahara, Touareg 